GPO PRICE $\$$
CFSTI PRICE(S) $\$$

Hard copy (HC) 3.00

Microfiche (MF)

.65

ff 653 July 65

SPACE SCIENCES DATA PROCESSING

\author{
BY \\ GEORGE H. LUDWIG
}

NASA

GODDARD SPACE FLIGHT CENTER

GREENBELT, MARYLAND

\author{
NOVEMBER 1966
}

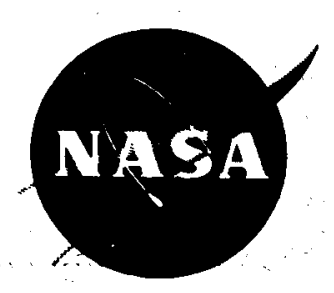

J 
$X-560-66-528$

SPACE SCIENCES DATA PROCESSING

By

George H. Ludwig

Presented at the 13th Nuclear Science Symposium in Boston, Massachusetts, October 1966. 
TABLE OF CONTENTS

$\underline{\text { Page }}$

ABSTRACT ........................ . . iii

INTRODUCTION . . . . . . . . . . . . . . . . 1

THE GENERALIZED INFORMATION SYSTEM. . . . . . . . . 1

ON-BOARD DATA PROCESSING AND REDUCTION • . . . . . . . 4

GROUND CENTRAL DATA PROCESSING . . . . . . . . . 5

ADDITIONAL GROUND DATA REDUCTION . . • . • • • • . 8

Data Scanning . . . . . . . . . . . . . . . . 9

Provide a Mapping in Either Time or Space. . . . . . . . . 9

Fully Analyze Especially Significant Portions of Data . • • . . 11

THE EXPERIMENTERS TIMETABLE . . . . . . . . . . . 11

CONCLUSION . . . . . . . . . . . . . . . . . . 14

REFERENCES . . . . . . . . . . . . . . . . . 14 


\begin{abstract}
Spacecraft carrying large numbers of scientific instruments are presently transmitting data at the rate of approximately 100 million data points per day. The outputs of the sensors are partially processed on the spacecraft and transmitted to the ground. These data must then be converted from raw digital form into a conceptually meaningful form which the experimenters can analyze and from which they can draw valid conclusions about the phenomena being measured. At present the ground processing is done in several steps. The first includes conversion of the raw data acquisition station tapes into computer tapes and includes signal cleanup, establishment of synchronization, and time decoding. In the newest processing lines this first step also includes a moderate amount of editing and quality control. The rest of the steps involve large scale computers and include further editing, establishment of accurate timing, computation of the spacecraft attitude, and sorting, to provide data tapes for the individual experimenters. The experimenters have the responsibility for the further reduction to more meaningful form. These operations include further sorting, storage, compilation, computation, and display. There is at present a great need for additional development of analysis and display programs, techniques, and equipment to assist in this work.
\end{abstract}




\section{SPACE SCIENCES DATA PROCESSING}

George H. Ludwig

\section{INTRODUCTION}

In the few years since the first spacecraft the information system capabilities have expanded greatly to meet the needs of the experimenters for more and more complex and discriminating measurements from their remote sensors. The number of spacecraft launches has increased steadily, and the average size, and therefore experiment payload weight, has also increased. As a result, we are now receiving a very large volume of space data. The end goal of all the data handling activities is to permit the experimenters to reach conclusions about the phenomena being measured by their instrument sensors. All on-board processing, coding, telemetry, decoding and ground processing are intermediate steps to that goal. But because of the distant location of the sensors and the data volume, these data handling operations pose complex technical and operational problems.

This paper discusses some of the information system problems from the standpoint of the experimenters who are the users of the system. The discussion is restricted to one class of spacecraft, the NASA managed and operated space sciences satellites. Department of Defense, applications (weather and communications), and deep space craft have their own special sets of problems and are not discussed here. The daily data volume from these GSFC space sciences satellites is approaching 150 reels of tape recordings (each 2500 feet in length) of the data acquisition receiver outputs. This corresponds to about 100 million data points per day, where a data point is a measurement of some quantity such as voltage, current, number of pulses in a given time, etc. As of 1 October 1966, these data are obtained from 14 operating spacecraft by a network of 12 data acquisition stations.

The term information system refers to the electronic systems which collect the outputs from the experiment sensors on the spacecraft, process these data on the spacecraft, transfer them to the ground receiving stations, and prepare the information in suitable form for the experimenters so that they may reach conclusions about the phenomena being measured.

\section{THE GENERALIZED INFORMATION SYSTEM}

A generalized information system for space experiments is illustrated in Figure 1. The sensors on the spacecraft are provided by the individual 


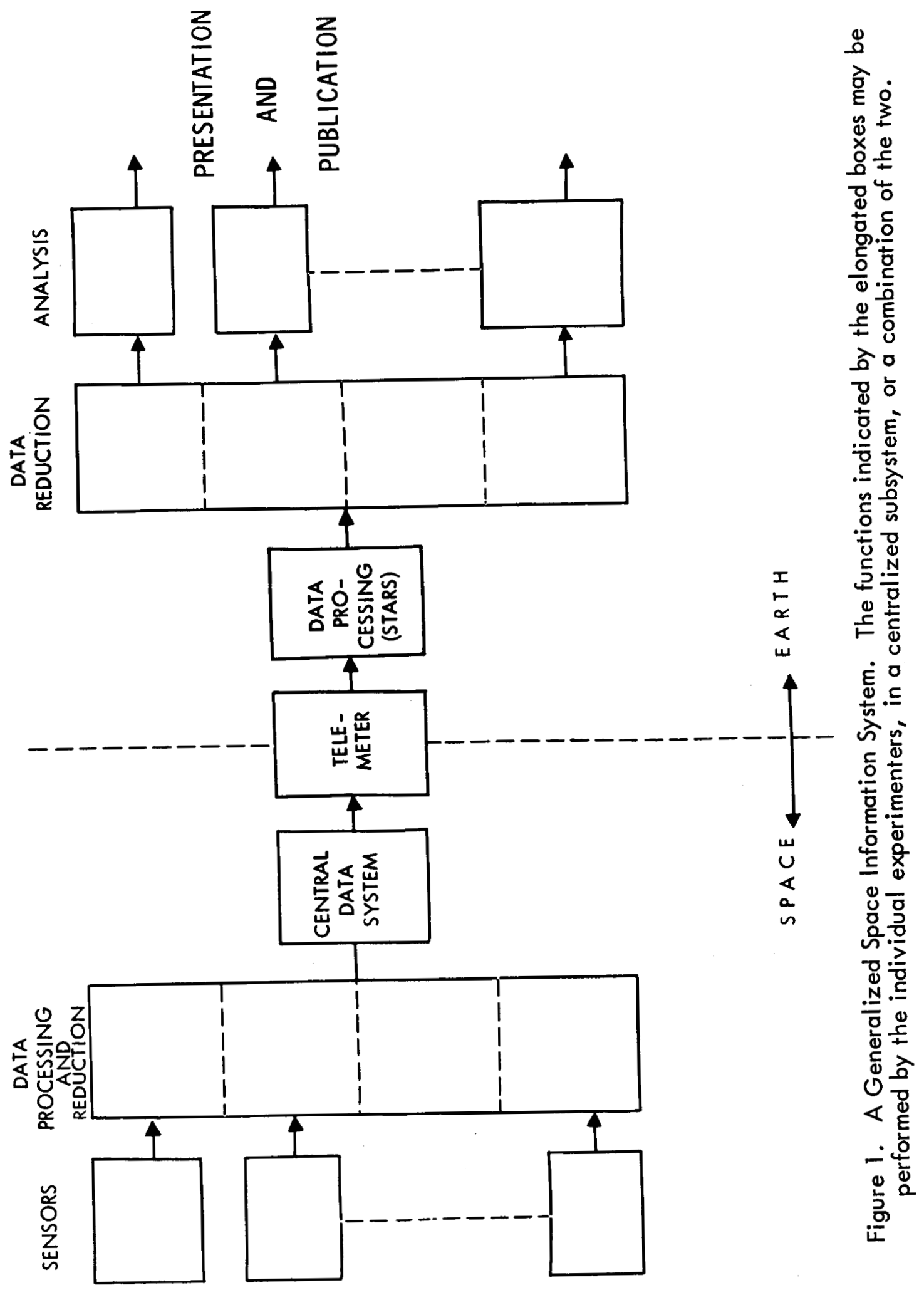


experimenters and convert physical quantities such as temperature, charged particle energy, magnetic field intensity, etc. into electrical quantities. Signal conditioning circuits aboard the spacecraft such as amplifiers, feedback networks, charge integrators, etc. are frequently associated directly with the sensors to make the electrical quantities more easily processed and telemetered. These are often followed by additional processing circuits to count pulses, measure the amplitudes of pulses, measure the amplitudes of more slowly varying analog quantities such as current, voltage and resistance, and to measure time intervals in order to simplify the task of telemetering the data to the ground. After this processing, additional processing of two basic types may be done on the spacecraft. The first employs processing to reduce the amount of raw data without reducing the information content. This includes the elimination of redundant information, the elimination of meaningless zero readings and the reformatting operations which involve simply the rearrangement of the data. The second type of on-board processing is that which reduces the information content of the raw data, including such processes as curve fitting, statistical analysis, mathematical manipulation, etc. These data processing and reduction operations on the spacecraft are shown in one elongated box in Figure 1 with dashed subdivisions. This signifies that these operations may occur either within the instrumentation provided by the individual experimenters or in a central processor.

The central data system indicated in Figure 1 includes those functions for gathering the data from all of the individual sources, frequently by means of a time-division multiplexer, the provisions for bulk data storage either for time scale conversion or to permit reception of data for extended periods by the use of a few localized receiving stations, and the data encoding functions. This is followed by the telemetry link which includes the transmitter, receiver, antennas and the space path. This entire link can be represented, in general, by a transfer function in which noise is added to the data.

After the data have reached the ground it is necessary to perform a number of additional operations before they can be used by the experimenters in their analyses. A number of these functions are normally performed in a central processing facility and include synchronization, noise removel, time decoding, quality determination, editing, and decommutation or sorting. These steps result in the provision to the experimenters or other users of data tapes containing the best estimation of the original data from each experiment output, along with the necessary status, performance, time and validity information. It is also common practice to supply orbit and spacecraft attitude information necessary for use in the experimenter's analyses.

These operations are followed by a number of additional processing steps in which the data are reformatted, sorted, stored, used in mathematical analyses and manipulations and which result in the provision of the data in a form 
which can be readily interpreted. This function, too, is shown gathered into one large block with dashed subdivisions, again indicating that this function is performed sometimes by the individual experimenters and sometimes in the central facility.

The final step is the analysis of the information received by the experimenters in order to ascertain some characteristics of the phenomena being investigated by the sensors. This analysis results in conclusions about the phenomena and the presentation and publication of these results.

The general data system described above is one way. It is frequently necessary, especially in the larger spacecraft, to convey instructions from the ground back to the spacecraft, either to control the routine operation of the spacecraft, or to adjust the experiments or other subsystems for best operation. This additional command link is conceptually the same as the system described above, with the direction of information flow directed from the ground back up to the spacecraft.

Three portions of this generalized data system will be discussed at greater length in this paper. They are the on-board data processing and reduction; the central data processing on the ground; and the data reduction which provides the necessary outputs for analysis.

\section{ON-BOARD DATA PROCESSING AND REDUCTION}

Thus far data processing on the spacecraft has been kept relatively simple in order to obtain high equipment reliability and high confidence in our ability to interpret the results after flight. However some steps have already been made in the direction of increasing the amount of data processing on board the spacecraft. A very simple example is the inclusion of floating point counters on several spacecraft which count pulses in a nonlinear manner. These counters have very large dynamic ranges and fixed accuracies which are ideal for many of the intensity and count rate measurements in a wide variety of experiments.

There are a number of arguments at this time for developing more extensive on-board processing techniques. A major argument is that the sheer volume of data being returned from the satellites is becoming extremely large and the task of processing the data on the ground both in the central facility and by the individual experimenters is expensive and time consuming. Another strong argument is that this may make it possible to obtain information that might not otherwise be possible because of telemetry link bandwidth limitations. A system designed for significant on-board data processing should be designed so that any degree of on-board processing from essentially zero to a larger amount can be 
performed by programming from the ground after the spacecraft has been launched, at least in the early systems. Thus, the spacecraft can be launched with a very simple data processing format and as the characteristics of the phenomena and the behavior of the instruments are ascertained in orbit, then additional degrees of data processing can be employed. On-board computers with stored but replaceable programs are now being developed by several groups. These computers may also have the ability to control the calibrations of the experiments, the computation of spacecraft attitude relative to the sun or magnetic field (with resulting control of experiment sampling times and programs), and may interface with the spacecraft attitude control, power, and thermal control subsystems. Any on-board processing system of this type should be designed so that it can at any time revert to a very simple method of processing the data to verify the proper operation of the experiments. The experimenters must at all times have a high level of confidence in the data, particularly if anomalous effects are seen.

\section{GROUND CENTRAL DATA PROCESSING}

Aftec the satellite telemetry data are recorded on tape recorders at the twelve data acquisition stations located around the world, these tapes are forwarded to the Goddard Space Flight Center for processing. The initial processing is performed in the central facility which includes the necessary production control, tape inventory, quality control, and library provisions, in addition to the processing lines and computer equipment. As was mentioned earlier, the output of this facility is a set of tapes for each experimenter containing the data from his experiment plus the necessary auxiliary information and the spacecraft orbit and attitude. The functions performed by this central data processing facility are illustrated in Figure 2 for the Orbiting Geophysical Observatory. 2 Similar functions are performed for the other scientific satellites. One tape from each shipment from each data acquisition station is evaluated soon after receipt at Goddard Space Flight Center. The purpose of this evaluation is to determine whether the equipment was operating properly at the data acquisition stations, in order that abnormalities can be corrected as soon as possible. After evaluation the tapes are mounted on the Satellite Telemetry Automatic Reduction System (STARS) for the initial processing operation. In these processing lines the signals are conditioned and bit word and frame synchronization are established. In the signal conditioning operation a best guess is made as to the original content of the signal on a bit-by-bit or tone burst-by-tone burst basis. This operation usually includes the integration of the signal for the duration of the bit or tone burst period before a decision is made. At the same time that these functions are occurring, the times recorded on the tapes at the data acquisition stations are decoded and continuously compared with a locally 


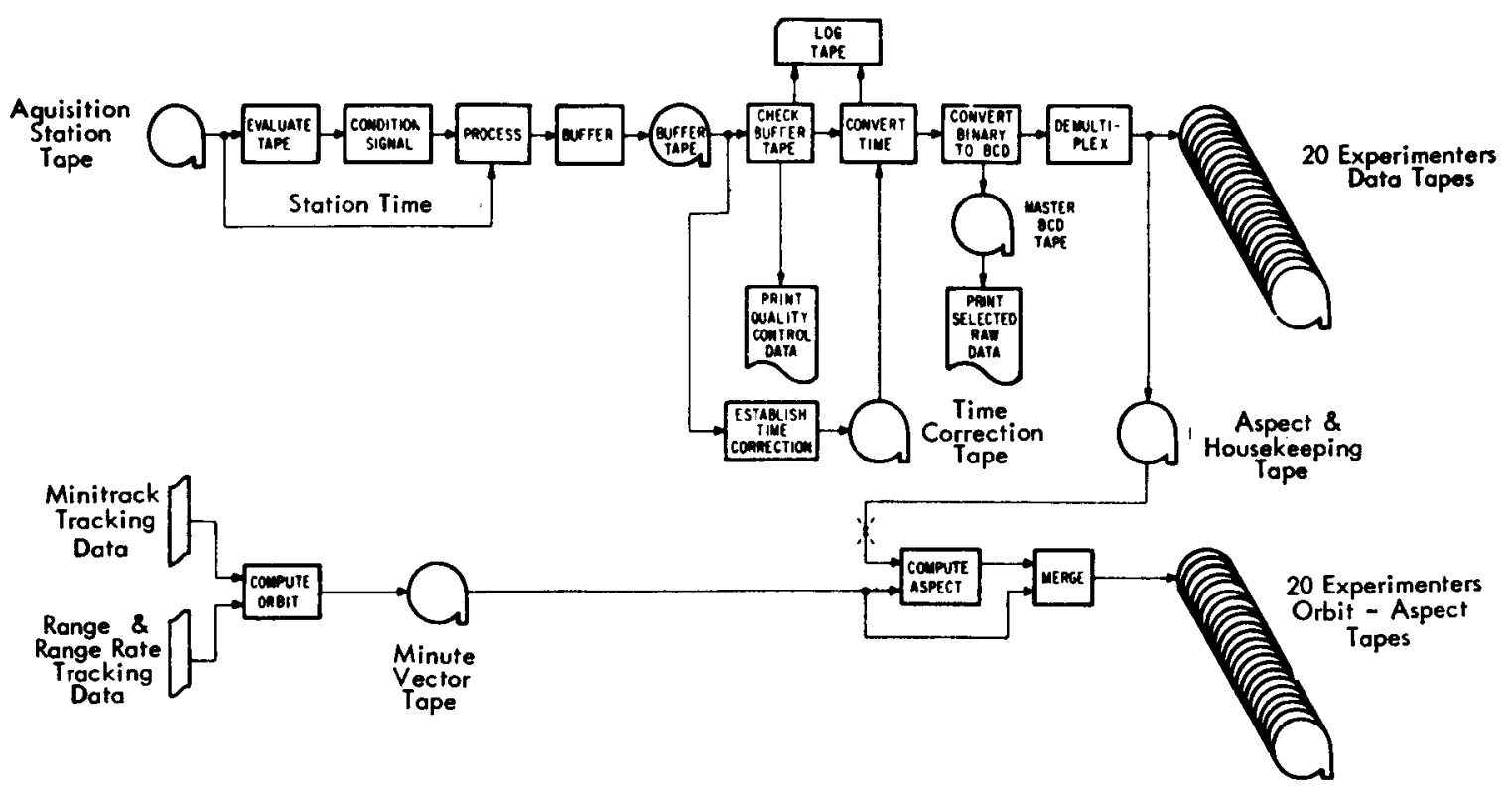

Figure 2. Data Processing Performed in the GSFC Central Facility for the Orbiting Geophysical Observatory. This is representative of the processing performed for all the GSFC scientific satellites.

running clock. Both the reconstructed telemetry data and the time are buffered in a core memory and then recorded on a computer compatible buffer tape.

In the latest model of the processing lines, referred to as STARS phase II, a CDC 3200 computer is included to perform two major functions. The setup of the processing line is under computer control to reduce the time required to prepare for each run, and to reduce the number of operator errors. Initial computer processing editing operations are performed during this first pass to determine the quality of the data as early as possible. In addition, a simulator is included for system checkout. A photograph of a STARS II PCM processing line is shown in Figure 3.

After the first processing operation on the STARS lines, all additional processing is performed on general purpose computers. The operations performed in these computers are also indicated in Figure 2. An editing check of the buffer tapes determines the internal consistency and the quality of the data on those tapes. The times, either telemetered with the data from the spacecraft clock, or recorded with the data from a ground clock, or both, are converted to Greenwich Mean Time (GMT). Corrections are made for clock errors and propagation times. This step is especially crucial, since it is common practice to use time to correlate the data with orbital position and with other housekeeping and experiment data during the analysis phase. 


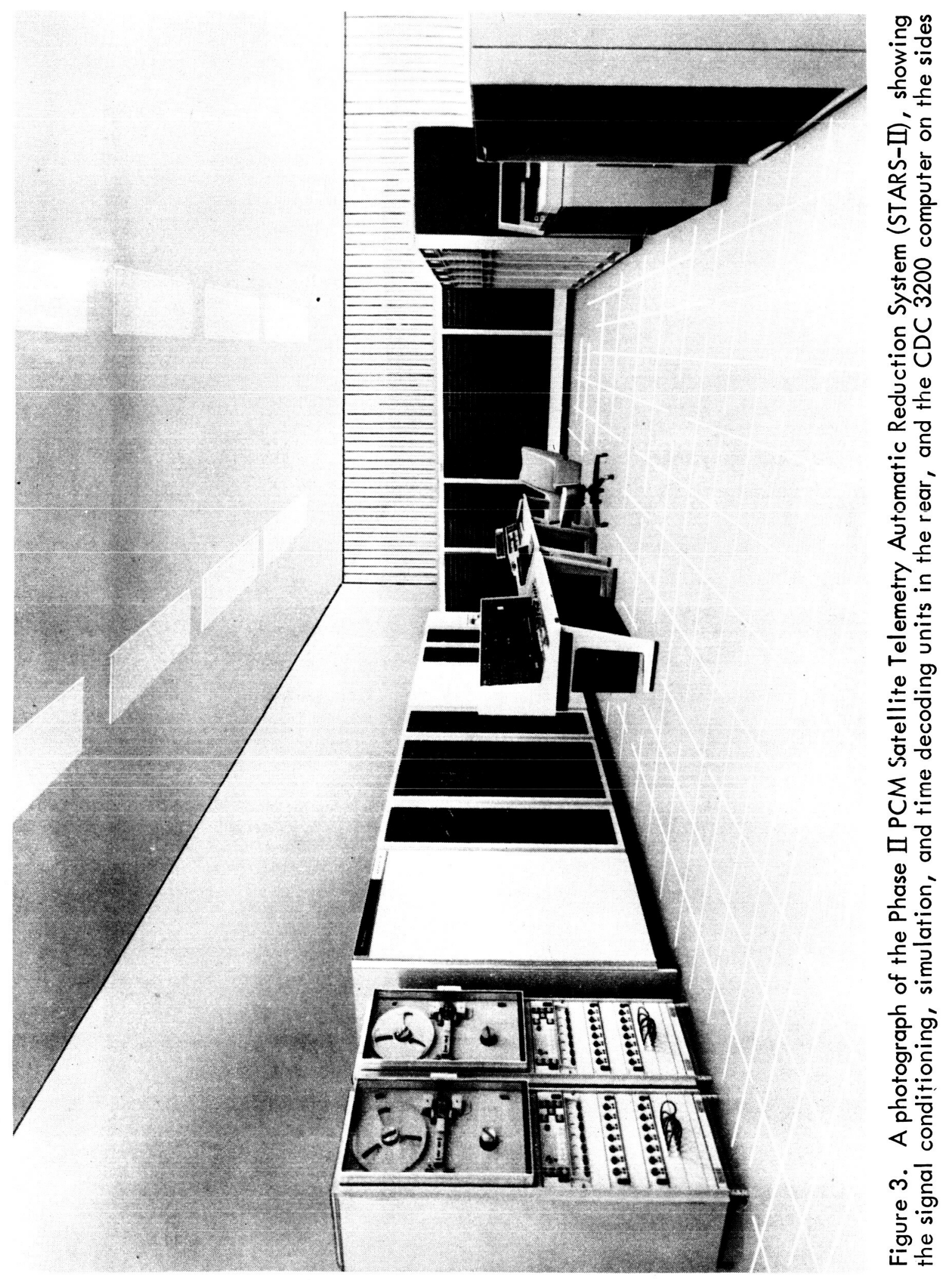


The data, now including GMT, are converted into a form which is convenient for the experimenters. Then they are sorted (decommutated) onto separate data tapes for each experimenter. Each tape contains the data from a specific experiment, GMT, and various pertinent spacecraft data, such as the temperature of the experiment mounting plate, the voltage of the power bus, etc.

In parallel with the telemetry data processing, the spacecraft orbit is computed for each minute using inputs from the radio interferometer and range and range rate tracking stations. This smoothed orbit, along with attitude control system error signals obtained from the telemetered spacecraft data, is used to compute the minute by minute look directions for the detectors. Both the orbit and attitude information are shipped to the experimenters along with their telemetered data.

In connection with the data processing operations outlined above, it is necessary to conduct a number of bookkeepings and library functions. The origina] data acquisition station tapes are cataloged when they are received and are eventually placed in permanent storage in the analog tape archive, which at present contains approximately 100,000 reels of tape. An intermediate digital tape referred to as the "edit tape" is also placed in permanent storage for use in the event that it is necessary to redo the computer processing. A number of documents are generated during the processing operation which are used by the processing facility staff to evaluate performance and by the experimenters so that they may have an accurate record of the content of all of the tapes and the quality of the data. Since the identification of each command received by the spacecraft is not contained in the telemetry data it is also necessary to decode the commands from the original acquisition station tapes where they are recorded at the time of command transmission. The complete command list is then furnished to the experimenters in the form of a magnetic tape, punched cards, or a listing. This task may be quite large by itself, since tens of thousands of commands are transmitted to the OGO's.

\section{ADDITIONAL GROUND DATA REDUCTION}

After the data are available in raw form, the experimenters are still faced with the monumental task of reducing these data into forms from which they can reach meaningful conclusions about the phenomena being investigated. This function commonly includes reformatting, sorting, merging, accumulation, statistical analysis, and mathematical manipulation of the raw data. It includes some provision for outputting the summarized data in a readable form such as line printer tabulations, $\mathrm{x}-\mathrm{y}$ plots, motion pictures, etc. 
In the performance of this activity it is sometimes necessary to provide data outputs for three different processes which may be performed for a particular experiment.

$\underline{\text { Data Scanning }}$

It may not be necessary to perform a detailed analysis of every piece of data received from a particular experiment. It is generally necessary in these cases to scan all of the data to select the interesting portions, since it will not be possible to predict the time periods or regions in space which will produce these interesting data. To illustrate, bursts of particles are frequently emitted by the sun. One of the objectives of many of the experiments is to detect these unpredictable bursts and analyze them in detail.

In order to avoid an extremely long and painful process in scanning all the data, it is common to reduce them to strip charts or some other visually meaningful form for rapid viewing. One of the most recent techniques which shows great promise for this function is the production of motion pictures generated by taking sequences of photographs of a suitable display of the data. In one case the amplitude of the output of a swept frequency very low frequency (VLF) receiver was plotted as a function of the frequency to which it was tuned to provide a frequency spectrum. ${ }^{3}$ A picture was taken of this spectrum each time the receiver sweep was completed, or once every 256 telemetry frames. When viewed in rapid succession by the use of a motion picture projector it is possible to quickly spot interesting fluctuations from the steady state condition.

\section{Provide a Mapping in Either Time or Space}

A frequent scientific objective is to determine the spatial extent of specified phenomena. Thus, it is necessary to take the data from a period during which the spacecraft moves from position to position in its orbit, and the location of the orbit moves in a sun-earth coordinate system. Thus, a complete mapping in space may require a year or more. It is then necessary to reduce these data to one or a few plots to provide a map of the phenomena. Figure 4 is an example of the compression of 6 months of data from the Interplanetary Monitoring Probe (IMP-I), in order to ascertain the over-all picture of the direction and strength of the magnetic field in the tail of the earth's magnetosphere. ${ }^{4}$ This single plot represents approximately $10^{5}$ individual data points. The direction of the magnetic field is indicated by the directions of the individual arrows, and the strength at each position is indicated by the lengths of these arrows. One can obtain a clear impression of the neutral plasma sheet which was discovered by Dr. Norman F. Ness and his co-workers from these data. It is located along the line where the field reverses its direction from outward directed to inward directed. 


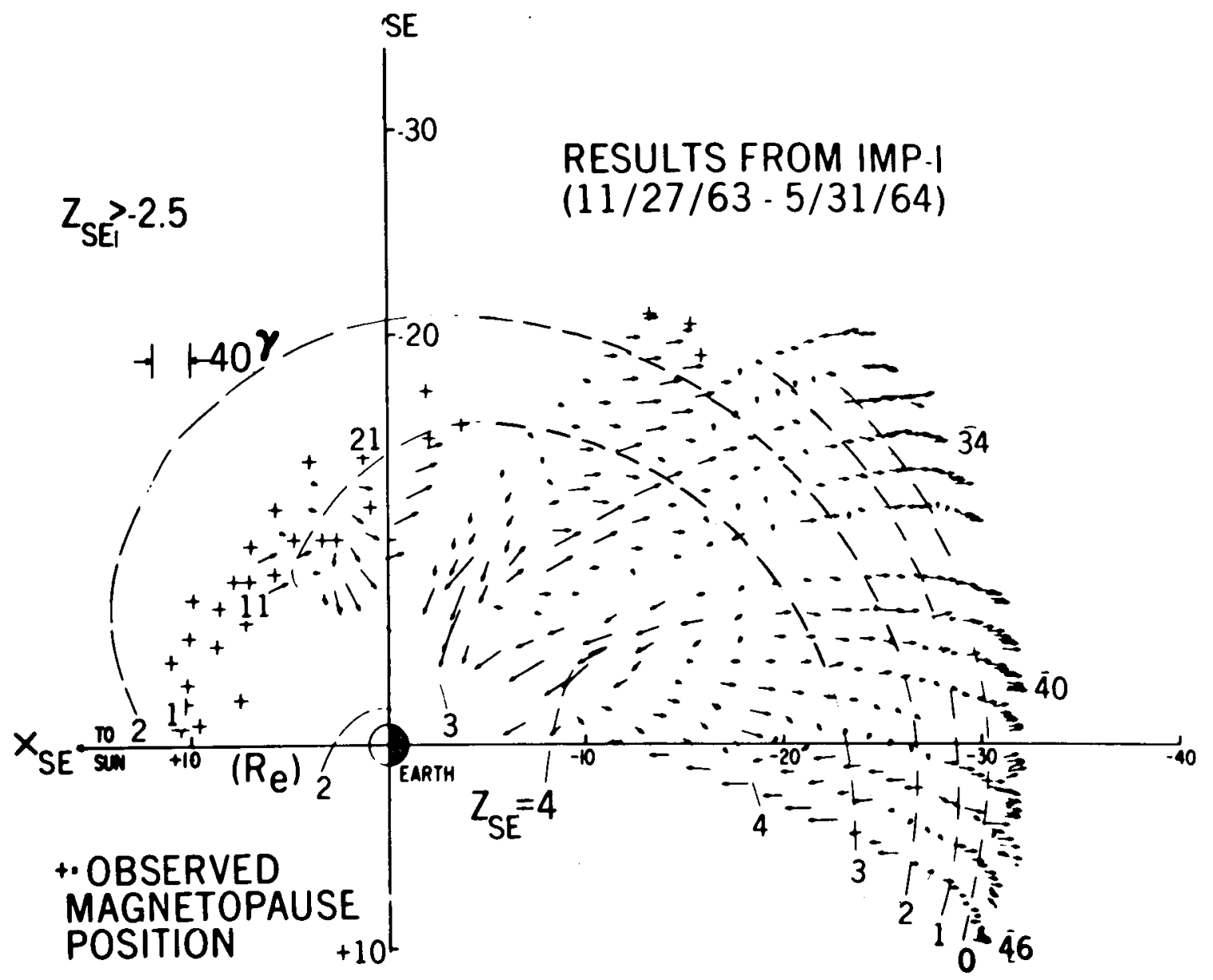

Figure 4. Projection of $X$ (solar ecliptic) - $Y$ (solar ecliptic) component of the magnetic field as measured by IMP - I onto the ecliptic plane when the satellite is above $Z$ ( solar ecliptic) = -2.5 earth radii. Crosses indicate traversal of the magnetospheric boundary; circles indicate orbit numbers. 4

In addition to the requirement for mapping in space it is also frequently necessary to provide a map in time, with scales ranging from minutes or hours to many years. For example, in studying numerous solar related phenomena, some changes occur in a few minutes during the buildup of a solar flare. On the other hand, other changes involve a time scale equal to the length of the sun spot cycle of eleven years. Thus, it may be necessary to investigate the data from a particular type of sensor for at least that period of time in order to have a complete understanding of the phenomenon. 


\section{Fully Analyze Especially Significant Portions of Data}

It may be possible in many cases to select a limited portion of the data for detailed analysis. This might include, for example, data obtained during transversal of the magnetospheric boundary surface, the transition region, and the shock front if one is studying the interaction of the solar plasma and the earth's magnetic field. Another example is the detailed study of the ionization layers surrounding the earth at heights of from one hundred to several hundred kilometers. Additional examples of interesting subjects for detailed study include solar storms, day-night effects, high altitude nuclear explosions, the auroral zone and the astronomical investigation of certain portions of the celestial sphere.

Some experimenters may require the full analysis of all of the data for extended periods of time. Most generally, this will be for those experiments which have event accumulation rates low enough to require data from long operating periods to obtain statistical significance. An example of this type of experiment is the cosmic ray experiment where it may take many months to accumulate a meaningful body of data. This is illustrated in Figure 6 in which the data from a low energy cosmic ray experiment on OGO-I are plotted for a 25 day period. ${ }^{5,6}$ Each point on the graph represents the detection of a single cosmic ray in the energy range from 20 to $80 \mathrm{Mev}$ per nucleon and particle species range from helium to iron. The instrument makes two independent measurements for each event, the rate of energy loss represented by $\triangle \mathrm{E}$, and the remainder of energy represented by $E-\triangle E$. In a plot of the type shown in Figure 5 the individual nuclear constituents are sorted by charge. Thus, helium, lithium, beryllium, boron, carbon, etc. can be identified on the plot. During this 25 day period enough events have been registered to show the carbon and oxygen lines quite clearly. However, in order to provide a meaningful measure of the amount of boron, nitrogen, and other rare elements it is necessary to accumulate data for many months.

Note that the processing techniques for meeting each of the three data analysis requirements listed above may be considerably different. Any one experiment may require processing programs for outputs for all three analysis functions. Thus, the experimenters processing requirements may be considerable.

\section{THE EXPERIMENTERS TIMETABLE}

Each experimenter invests a considerable effort in the performance of a specific experiment. The representative timetable shown in Figure 6 indicates that a specific experiment may require a period of from seven to nine years 


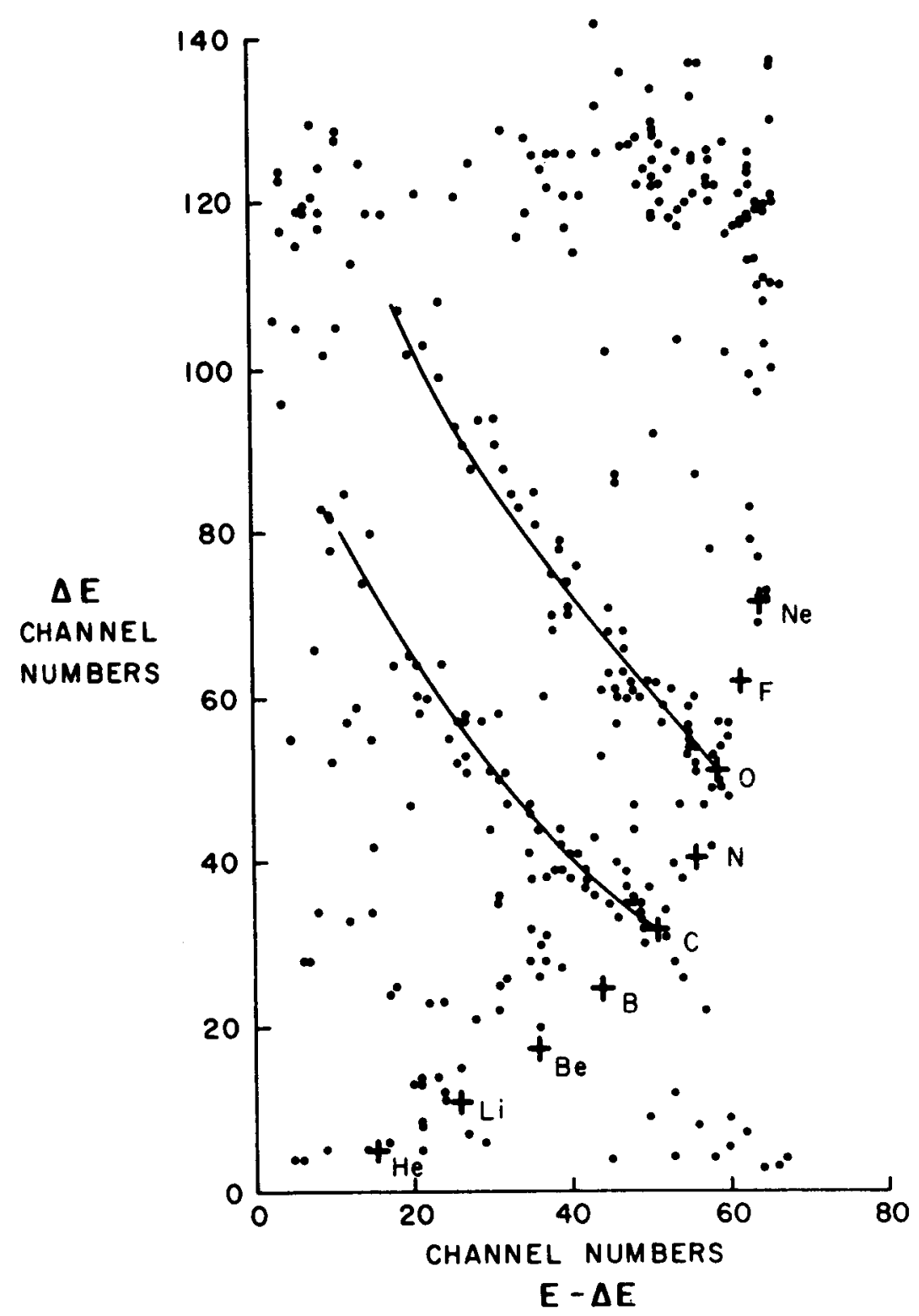

OGO-I, 25 OCT. - 19 NOV. 1964 HIGH $Z$ MODE

Figure 5. Medium and High Charge Cosmic Ray Events Measured by a Nuclear Abundance Detector on OGO-1.6 The rate of energy loss is nearly proportional to $\triangle E$, the energy lost in a thin scintillator, while $E-\triangle E$ is nearly proportional to the remainder of the particle energy. 5 These two measurements permit unambiguous determination of the mass and initial energy of each cosmic ray. 
for its completion, including the time from initial conception of the experiment to completion of the work on the data. Even before the experiment selection, the experimenter may have invested a considerable effort in the development of a new detector technique. He commonly builds the instrumentation for a specific mission during the period from one to three years before the launch. Preparation for the data processing activities sometimes begins as early as two to three years before launch. However it must be kept in mind that the experimenter is frequently not able to predict the detailed characteristic of his expected data accurately enough to permit him to write the final processing programs before launch. It is often necessary for him to study the actual flight data before he can decide the exact manner in which he will process the data and present it for analysis. Thus, after launch it is common for an experimenter to require up to one year for the preparation of the final processing programs. His detailed analysis occurs over a period of from two to four years.

\section{SELECTION}

Build Instruments

Prepare Data Plan

Complete Preliminary

Programs

Present Preliminary Results

Complete Final Programs

Data to Others

Present Final Results

Archival

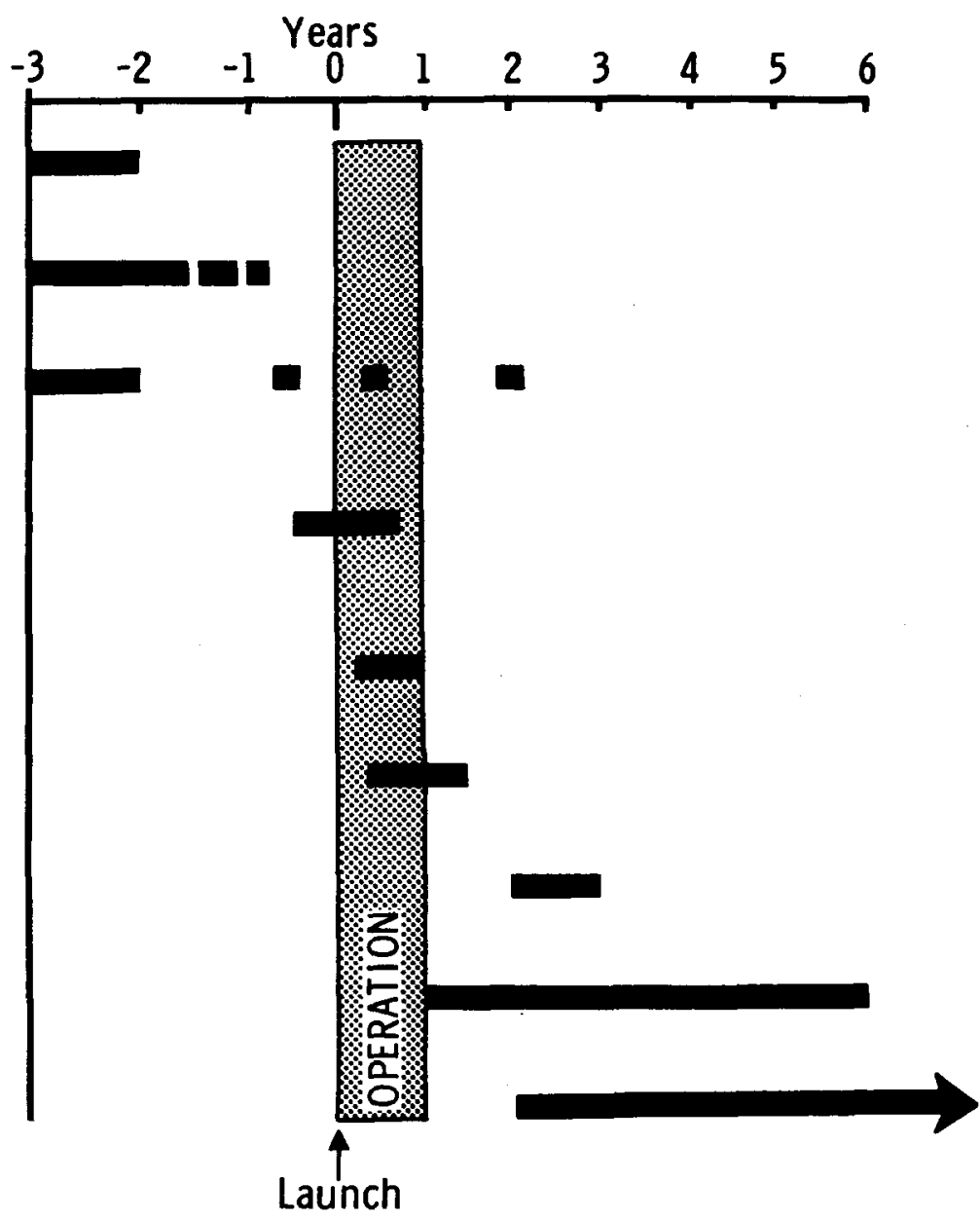

Figure 6. A Typical Time Scale for the Conduct of Space Science Experiments 
In view of this large investment in time and effort by the experimenters, it is becoming increasingly important to investigate new and faster methods for the preparation of programs and presentation techniques. In addition, the efficient use of on-board processing devices should also eventually assist in the shortening of the experimenters workload and period of analysis.

\section{CONCLUSION}

The processing of data from NASA's scientific satellites is a monumental task involving millions of dollars worth of equipment, programming effort, and operating manpower. The cost for processing the data from a scientific experiment frequently exceeds the cost of developing and building the instruments for that experiment. Thus, considerable effort is being expended and must continue to be expended to make this processing more efficient and productive. For this purpose, advanced techniques for processing the data both on the spacecraft and on the ground, and for the presentation of these data in meaningful form to the experimenter for scanning and analysis are under development at a number of field centers within the NASA.

\section{REFERENCES}

1. Bostrom, C. O. and G. H. Ludwig, "Instrumentation for Space Physics," Physics Today 19, p. 43 (July 1966)

2. Ludwig, G. H., "The Orbiting Geophysical Observatories," Space Science Reviews 2, p. 175 (1963)

3. Ficklin, B. P., Stanford Research Institute, private communication

4. Ness, N. F., "The Earth's Magnetic Tail," Journal of Geophysical Research 70, p. 2989 (1965)

5. Bryant, D. A., G. H. Ludwig, and F. B. McDonald, "A Scintillation Counter Telescope for Charge and Mass Identification of Primary Cosmic Rays," IRE Trans. on Nuclear Science, NS-9, p. 376 (1962)

6. Balasubrahmanyan, V. K., D. E. Hagge, G. H. Ludwig, and F. B. McDonald, "The Multiply Charged Primary Cosmic Radiation at Solar Minimum, 1965," Journal of Geophysical Research 71, p. 1771 (1966) 